\title{
Volumineux lipome intra-jugal : présentation d'un cas
}

\section{Tabeti-Bentahar CF, Oufriha N, Dahmani I, Bouzouina F. (Service de Pathologie et de Chirurgie buccales, CHU Oran-Algérie)}

Les lipomes sont des tumeurs bénignes conjonctives composées d'adipocytes matures, généralement entourées par une fine capsule fibreuse. Ce sont les plus communes des tumeurs des tissus mous et environ 15 à $20 \%$ des cas sont retrouvés dans la région de la tête et du cou. Cependant, seulement $1 \%$ à $4 \%$ des cas apparaissent au niveau de la cavité buccale [Aguiar de Freitas et al. 2009, Allon et al. 2011]. Le cas rapporté est celui d'un homme âgé de 59 ans, reçu à la consultation du Service de pathologie et Chirurgie Buccales pour une tuméfaction de la joue gauche évoluant depuis plusieurs années, de consistance rénitente, indolore à la palpation, non adhérente par rapport au plan cutané et d'évolution progressive. L'échographie a retrouvé une formation nodulaire hypoéchogène, hétérogène de 34,8 x 18,3 mm, peu vascularisée en écho doppler, sous muqueuse, sans paroi propre. L'examen tomodensitométrique a révélé une formation hypodense grossièrement ovalaire de $37 \times 27 \mathrm{~mm}$, non réhaussée après injection de produit de contraste et sans signe d'envahissement. Après exérèse, l'examen macroscopique de la totalité de la pièce opératoire a permis de mettre en évidence le caractère encéphalloïde et les dimensions réelles de la lésion $(80 \times 30 \mathrm{~mm}$ ). L'examen anatomopathologique est revenu en faveur d'un lipome aux limites d'exérèse suffisantes; avec une lésion tumorale bénigne d'architecture lobulaire renfermant des cellules adipeuses régulières délimitées par des cloisons conjonctivo-vasculaires.

Les lipomes de la cavité orale représentent $0,5 \%$ à $5 \%$ de l'ensemble des tumeurs buccales (Bandéca et al. 2007). Dans la cavité buccale, ils surviennent le plus souvent isolément dans la région du plancher buccal, des joues, du vestibule, du palais, des gencives et de la langue (Hamisch et al. 2007). Leur croissance est lente et indolore ; leur couleur jaune (souvent jaune d'œuf) est parfois visible à travers la muqueuse, lorsque celle-ci est tendue. Lorsqu'elles sont situées en profondeur, ces tumeurs peuvent être inter ou intramusculaires, et rarement périneurales (Hamisch et al. 2007). Leur taille varie de 1,5 à $2 \mathrm{~cm}$, et leur consistance peut être ferme ou élastique, en fonction du tissu conjonctif qu'elles hébergent. Sur le plan histologique, les lipomes intra-oraux sont répartis en six sous types d'après Seifert 2000 : les lipomes simples, les fibrolipomes, les angiolipomes, les lipomes à cellules fusiformes, les angiomyolipomes et les hibernomes. D'autres formes de transition ont été décrites (avec des composantes myxomateuses ou kystiques, voire des zones d'ossification (Hamisch et al. 2007) ; en 2001, Nagal et al. ont décrit une nouvelle entité « les sialolipomes » : lésion associant du tissu glandulaire et des composants adipeux (Aguiar de Freitas et al. 2009). Le diagnostic différentiel des lipomes se fait avec le liposarcome où parfois, le recours à l'immunohistochimie est impératif car la distinction peut être difficile. La discussion diagnostique se fait aussi avec les tumeurs et pseudotumeurs glandulaires ; avec les tumeurs vasculaires et les tumeurs nerveuses. Le traitement des lipomes consiste en une exérèse simple (Bandéca et al. 2007).

Ce sont l'indolence et l'absence de gêne fonctionnelle qui sont à l'origine de telles tumeurs de volume impressionnant rencontrées jusqu'à l'heure actuelle sous nos latitudes.

TABETI-BENTAHAR Chérifa Faïza tabeti_cherifa@yahoo.fr 